ORIGINAL PAPER

\title{
KLK6 as a Predictor of Ovarian Malignancy
}

Primandono PERBOWO', Brahmana ASKANDAR ${ }^{1}$

\begin{abstract}
Objective: To measure the levels of KLK6 and CA 125 tumor marker and compare them as a predictor of epithelial type ovarian malignancy. KLK6 and CA125 were taken from blood serum of 60 ovarian tumor patients who met the inclusion criteria in consecutive sampling. CA125 quantitative examination and KLK6 titer were performed by ELISA. Surgical tissue from Dr. Soetomo Hospital, Surabaya was examined histopathologically at the Anatomical Pathology Laboratory of the Faculty of Medicine Airlangga University, Surabaya. Methods: This study is a diagnostic test using a cross-sectional design with a total sample of 60 ovarian tumor patients from obstetrics and gynecology outpatient clinic, Soetomo Hospital, Obstetrics and Gynecology Department, Faculty of Medicine, Airlangga University, Surabaya. CA125 was examined by ELISA, while KLK6 was examined using an ELISA reagent kit from Enzo Life Sciences Inc., USA. Results: This study examined a total sample of 60 patients, 30 patients (50\%) with benign ovarian tumors, 26 patients (43.33\%) with stage I ovarian cancer and 4 patients (6.66\%) with stage II ovarian cancer. The best cut off for KLK6 is $3.14 \mathrm{ng} / \mathrm{mL}$ and the standard CA 125 cut-off is $35 \mathrm{U} / \mathrm{mL}$. The sensitivity and specificity of Ca125 are 70.00; 33.33, the sensitivity and specificity of KLK6 are 50.00; 100.00, PPV and NPV of Ca1 25 are 51.22; 52.63, PPV and NPV of KLK6 are 100.00; 66.67. Conclusion: KLK6 showed high specificity for the diagnosis of ovarian cancer. This can improve the diagnostic accuracy of Ca125 which has high sensitivity but low specificity. A combined panel of Ca125 and KLK6 showed high diagnostic efficiency for early-stage ovarian cancer.
\end{abstract}

Keywords: kallikrein related peptidase 6 (KLK6), ovarian cancer, diagnosis, meta-analysis.

${ }^{1}$ Department of Obstetrics and Gynecology, Division of Gynecologic Oncology, Airlangga University, Indonesia
Corresponding author.

Primandono PERBOWO, Department of Obstetrics and Gynecology, Division of Gynecologic Oncology, Airlangga University, Indonesia.

E-mail: drprimandono28@gmail.com 


\section{INTRODUCTION}

Despite advances in therapy, ovarian cancer remains the most deadly gynecological cancers. Less than 30\% of women with advanced stages survive in the long term. When diagnosed in stage I, up to $90 \%$ of patients can be cured by conventional surgery and chemotherapy. At present, only $25 \%$ of ovarian cancers are detected in stage I partly because there are no specific symptoms and less effective screening strategies.

Given the prevalence of ovarian cancer and the importance of early detection to get a good prognosis, screening for early detection must have high sensitivity at an early stage ( $>75 \%)$, and very high specificity $(99.6 \%)$ to achieve a positive predictive value $>10 \%{ }^{1}$. Screening which is commonly used now, Ca125 and pelvic ultrasonography, has limited efficacy. In that case, one biomarker as parameters for early detection, screening and knowing disease progression is not enough and has to be more specific ${ }^{2}$.

The prognosis of cancer is determined by the ability to invade and metastasize. Metastasis is associated with invasive behavior of tumor cells in which cell membrane proteins, receptors, and extracellular matrix proteins play an important role. An important component in the protein regulation is proteases, an enzyme which plays a role in protein breakdown and catalyzes hydrolysis reactions. Hydrolysis reaction is a reaction involving water elements in specific substrate bonds, catalyzing the degradation of the interstitial matrix and basement membrane, allowing cancer cells to invade locally and metastasize to distant places. If protease is involved in the spread of cancer, it will have the potential to become new prognostic marker in cancer ${ }^{3}$.

In human body, protease that is widely expressed in ovarian cancer cells that play a role in cancer progression and metastases is kallikrein $(\mathrm{KLK})^{4}$. It is known that the family of kallikrein gene in human tissue contains at least 15 genes. All genes have important similarity, which are mapping at the same chromosome locus (19q13.4), good significant homology in both nucleotides and proteins, and the same genome arrangement. All genes encode a putative serine protease. It is known that some of the kalikrein genes are related to malignancy ${ }^{5}$. Kalikrein is expressed in many tissues, including tissues that produce steroid hormones or tissue-dependent hormones such as prostate, breast, ovary, and testicles. Most of kallikreins are regulated by steroid hormones in cancer cells $s^{5}$. Kallikrein is expected to act as a biomarker, indicator of disease progression, and control the invasive behavior of epithelial type ovarian cancer in in vivo and in vitro metastases ${ }^{6}$.

Such evidences clearly connect kallikrein and cancer development so that it can be applied as a new biomarker for the diagnosis and management of epithelial type ovarian cancer ${ }^{7}$. Each biomarker and panel from kallikrein family can predict response to chemotherapy, overall survival, short term survival (1 year survival rate), survival (tumor free progression) of ovarian cancer patients treated with chemotherapy ${ }^{8}$.

With these various potentials, it is expected that KLK6 can match the sensitivity and specificity of Ca125 which has been the gold standard of epithelial type ovarian cancer malignancy parameter. Moreover, Ca125 still has weaknesses, such as it can be applied to recurrent ovarian cancer, cancer persistency even though normal $\mathrm{Ca} 125$, and will not necessarily improve patient survival ${ }^{9}$. Thus, if KLK6 has very good sensitivity and specificity, and cost-effective, it can be used as a parameter of malignancy. Moreover, KLK6 also has the potential as the target of epithelial ovarian cancer therapy ${ }^{10}$. Therefore, this study was conducted with the aim of testing the accuracy, sensitivity, and specificity of kallikrein-6 (KLK6) by comparing it with Ca125 at Dr. Soetomo Hospital, Surabaya. By knowing those things, hopefully KLK6 can be used as an accurate biomarker for epithelial type ovarian cancer which exceed or at least match with $\mathrm{Ca} 125$. The combination of both therapies will provide more benefits, especially for early detection of epithelial type ovarian cancer so that it can improve the patient's survival time and quality of life.

\section{MATERIALS AND METHODS}

This study was a diagnostic test using a cross-sectional design with a total sample of 60 ovarian tumor patients from obstetrics and gynecology outpatient clinic in Dr. Soetomo Hospital, Obstetrics and Gynecology Department, Faculty of Medicine Airlangga University, Surabaya from June 2016 to March 2017. Blood sampling was taken before surgery for CA125 examination by ELISA, KLK6 was examined using ELISA reagent kit Enzo Life Sciences Inc., USA, measurement of Microplate Reader Biorad model 680 (bio-rad Laboratories Inc., USA) with Microplate Manager software version 5.2.1 and histopathology examination of the tumor tissue in the Pathology Anatomy Laboratory of Faculty of Medicine Airlangga University/Dr. Soetomo Hospital, Surabaya.

This research data was recorded in a special designed data collection form. Statistical analysis was conducted 
to see differences in serum KLK6 level between patients with benign ovary tumors and early stage ovarian cancer (stage I-II); the difference in tumor marker sensitivity and specificity; and the correlation between CA125 and KLK6. SPSS software was used to simplify the statistical analysis.

The inclusion criteria were patients who were diagnosed with ovarian tumors and had undergone surgery at RSUD Dr. Soetomo, had never been diagnosed with malignancy in the field of gynecology, and were willing to be the subject.

Exclusion criteria were non-epithelial ovarian cancer patients, stage III epithelial type ovarian cancer patients with or without ascites, and patients refusing to participate as the subject.

Predictors in this study were seen based on comparison of sensitivity, specificity, Positive Predictive Value, and Negative Predictive Value between KLK6 and CA125. The result of serum CA125 levels with an immune assay examination above $35 \mathrm{U} / \mathrm{mL}$ indicates a suspicion of malignancy. The titer result of serum KLK6 level with an immune assay examination above or equal to $3.14 \mathrm{ng} / \mathrm{mL}$ indicates suspicion of malignancy. CA125 sensitivity is the number of ovarian cancer patients with serum CA125 level above $35 \mathrm{U1} / \mathrm{mL}$ divided by the total number of ovarian cancer patients multiplied by $100 \%$. CA125 specificity is the number of benign ovarian tumor patients with serum CA125 levels below $35 \mathrm{U} / \mathrm{mL}$ divided by the total number of patients with benign ovarian tumors multiplied by 100\%. CA125 Positive Predictive Value is the number of ovarian cancer patients with serum CA125 levels above $35 \mathrm{U} / \mathrm{mL}$ divided by the total number of patients with serum CA125 levels above $35 \mathrm{U} / \mathrm{mL}$ multiplied by $100 \%$. CA125 Negative Predictive Value is the number of benign ovarian tumor patients with serum CA125 levels below $35 \mathrm{U} / \mathrm{mL}$ divided by the total number of patients with serum CA125 levels below 35 $\mathrm{U} / \mathrm{mL}$ multiplied by $100 \%$. The sensitivity of KLK6 is the number of ovarian cancer patients with titer serum KLK6 levels above or equal to $3.14 \mathrm{ng} / \mathrm{mL}$ divided by the total number of epithelial cancer patients multiplied by $100 \%$. The specificity of KLK6 serum is the number of benign ovarian tumor patients with titer serum KLK6 levels below $3.14 \mathrm{ng} / \mathrm{mL}$ divided by the total number of patients with benign ovarian tumors multiplied by $100 \%$. KLK6 Positive Predictive Value is the number of ovarian cancer patients with titer serum KLK6 level above or equal to $3.14 \mathrm{ng} / \mathrm{mL}$ divided by the total number of patients with titer serum KLK6 levels above or equal to $3.14 \mathrm{ng} / \mathrm{mL}$ multiplied $100 \%$. KLK6 Negative Predictive Value is the number of patients with benign ovarian tumors with titer serum KLK6 levels below $3.14 \mathrm{ng} / \mathrm{mL}$ divided by the total number of patients with titer serum KLK6 levels below $3.14 \mathrm{ng} / \mathrm{mL}$ multiplied by $100 \%$.

Statistical analysis was conducted using MEDCALC software. Statistical calculations used a significance level of $\mathrm{p}=0.05$ (5\%). It would be significant if $p<0.05$, on the contrary, if $p>0.05$, it was interpreted as insignificant. The researcher used the Receiver Operating Characteristic (ROC) curve to measure the performance of tumor markers. The area under the ROC curve of CA-125 (0.447) was lower than KLK6 (0.737). AUC approaching 1.0 indicates that the test has almost perfect discrimination, while AUC near 0.5 indicates poor discrimination.

\section{RESULTS}

The results obtained a total sample of 60 patients, 30 patients $(50 \%)$ with benign ovarian tumors, 26 patients $(43.33 \%)$ with stage I ovarian cancer and 4 patients (6.66\%) with stage II ovarian cancer.

The mean age of the healthy patient was 45.1 years, stage I ovarian cancer group was 49.3 years, stage III ovarian cancer group was 49.7 years. The statistical analysis of the three groups showed a normally distributed data and the comparison was significant $(\mathrm{p}=$ 0.027).

Table 5.1 showed the mean age in the cancer patients group was 46.3 years and the benign tumor group was 49.3 years. Based on the statistical analysis using T-test 2 free samples, the data was normally distributed but was not significant $(p=0.907)$.

The mean KLK6 in the healthy patient was 1.62 $\mathrm{ng} / \mathrm{mL}$, stage I ovarian cancer group was $12.05 \mathrm{ng} /$

Table 1. Age distribution of patients in the ovarian cancer and non-ovarian cancer groups (benign ovarian tumors)

\begin{tabular}{|l|c|c|c|}
\hline \multicolumn{3}{|c|}{ CaOvarium } & No \\
\hline Age & Yes & $46.0 \pm 12.4$ & P value \\
\hline$* t-2$ free sample test & $46.3 \pm 9.4$ & & 0.907 \\
\hline
\end{tabular}


Table 5.2. Distribution of CA125 and KLK6 levels in ovarian cancer

\begin{tabular}{|c|c|c|c|c|c|}
\hline \multicolumn{6}{|c|}{ CaOvarium } \\
\hline \multirow[t]{13}{*}{ KLK6 } & & Yes & No & Mc. Nemar & Kappa \\
\hline & $\geq 10.79$ & 1 & 0 & \multirow[t]{2}{*}{$<0.0001$} & \multirow[t]{2}{*}{0.313} \\
\hline & $<10.79$ & 29 & 30 & & \\
\hline & $\geq 6.0$ & 7 & 0 & \multirow[t]{2}{*}{$<0.0001$} & \multirow[t]{2}{*}{0.005} \\
\hline & $<6.0$ & 223 & 30 & & \\
\hline & $\geq 4.2$ & 13 & 0 & \multirow[t]{2}{*}{$<0.0001$} & \multirow[t]{2}{*}{$<0.0001$} \\
\hline & $<4.2$ & 17 & 30 & & \\
\hline & $\geq 3.14$ & 15 & 0 & \multirow[t]{2}{*}{$<0.0001$} & \multirow[t]{2}{*}{$<0.0001$} \\
\hline & $<3.14$ & 15 & 30 & & \\
\hline & $\geq 2.0$ & 18 & 10 & \multirow[t]{2}{*}{0.832} & \multirow[t]{2}{*}{0.036} \\
\hline & $<2.0$ & 12 & 20 & & \\
\hline & $\geq 1.5$ & 24 & 16 & \multirow[t]{2}{*}{0.052} & \multirow[t]{2}{*}{0.026} \\
\hline & $<1.5$ & 8 & 14 & & \\
\hline \multirow[t]{2}{*}{ Ca 125} & $\geq 35$ & 21 & 20 & \multirow[t]{2}{*}{0.061} & \multirow[t]{2}{*}{0.781} \\
\hline & $<35$ & 9 & 10 & & \\
\hline
\end{tabular}

$\mathrm{mL}$, and stage III ovarian cancer group was $110.79 \mathrm{ng}$ $/ \mathrm{mL}$. Based on the statistical analysis comparisons of the three groups, data was not normally distributed but significant $(p=0.001)$.

The mean CA-125 in the healthy patient was 15.37 $\mathrm{U} / \mathrm{mL}$, stage I ovarian cancer group was, $125.41 \mathrm{U} /$ $\mathrm{mL}$, and stage III ovarian cancer group was $1021.80 \mathrm{U}$ / $\mathrm{mL}$ with statistical analysis comparison of the three groups found that the data were not normally distributed and the results were significant $(\mathrm{p}=0.001)$.

Table 5.2. showed various types of cut-offs of KLK6, it turned out that the results were the same as other studies, which was able to predict ovarian malignancy at a cut off of $3.14 \mathrm{ng} / \mathrm{mL}(\mathrm{p}<0.05)$.

Table 3 below showed the examination of single CA-125 tumor markers had the highest sensitivity (70.00\%) compared to KLK6 with a cut off of $3.14 \mathrm{ng} /$ $\mathrm{mL}(50.00 \%)$. The highest specificity was possessed by KLK6 with a cut off of $3.14 \mathrm{ng} / \mathrm{mL}$ (100\%) both used as a single tumor marker examination or in combinati- on while compared to the CA-125 (33.33\%) as a standard.

Figure 1 showed the KLK6 accuracy in determining ovarian cancer was 0.737 .

Figure 2 showed that the accuracy of CA125 in determining ovarian cancer was 0.447 .

\section{DISCUSSION}

Ovarian cancer is the biggest cause of cancer-related deaths in women in Western Europe and the United States, and has the highest mortality rate of all gynecological malignancies. The mean age of early ovarian cancer patients is around $37.2-55.7$ years $^{10}$. In this study, the mean age was 46.3 . The pre-cancer to insitu cancer stage age was 37.2 - 46.3 years (Table 1 ). Another study from Egypt by Sherbini et al. reported that the mean age of advanced ovarian cancer incidence was 59.8 year, so it can be concluded that at the age of 45 years or at the pre menopause stage, tumor marker

Table 3. CA-125 and KLK6 Sensitivity, specificity, positive predictive value and negative predictive value

\begin{tabular}{|c|c|c|c|c|c|c|c|}
\hline & Degree & Sensitivity & Specificity & NPP & NPN & LR+ & LR- \\
\hline \multirow[t]{6}{*}{ KLK 6} & 10.79 & 3.33 & 100.00 & 100.00 & 50.85 & - & 0.97 \\
\hline & 6.0 & 23.33 & 100.00 & 100.00 & 56.60 & - & 0.77 \\
\hline & 4.2 & 43.33 & 100.00 & 100.00 & 63.83 & - & 0.57 \\
\hline & 3.14 & 50.00 & 100.00 & 100.00 & 66.67 & - & 0.50 \\
\hline & 2.0 & 60.00 & 66.67 & 64.29 & 62.50 & 1.80 & 0.60 \\
\hline & 1.5 & 80.00 & 46.67 & 60.00 & 70.00 & 1.05 & 0.43 \\
\hline Ca 125 & 35 & 70.00 & 33.33 & 51.22 & 52.63 & 1.05 & 0.90 \\
\hline
\end{tabular}




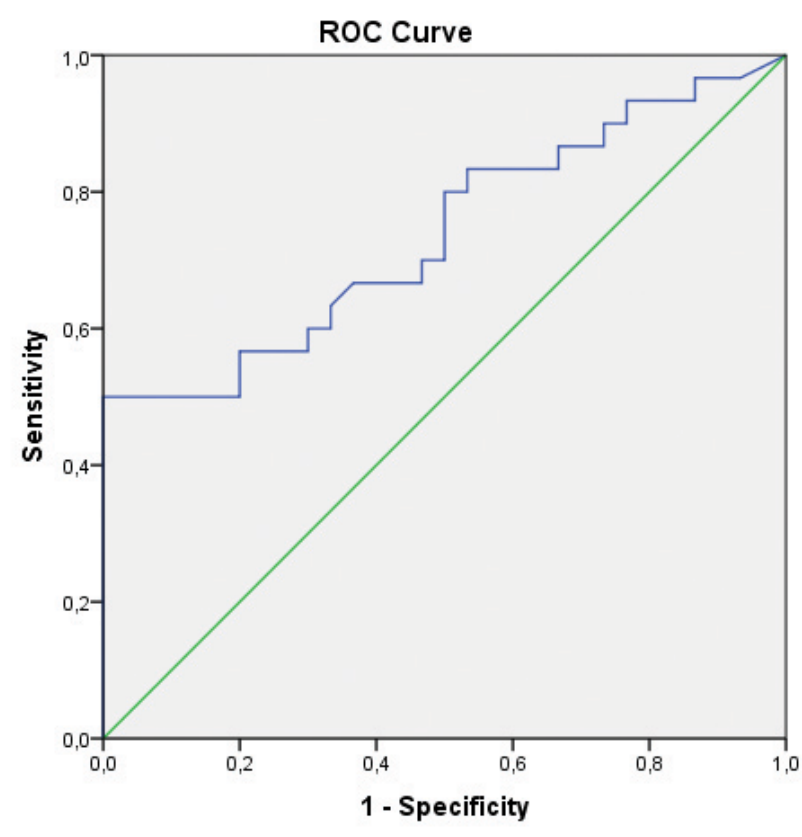

Diagonal segments are produced by ties.

Figure 1. ROC from KLK6.

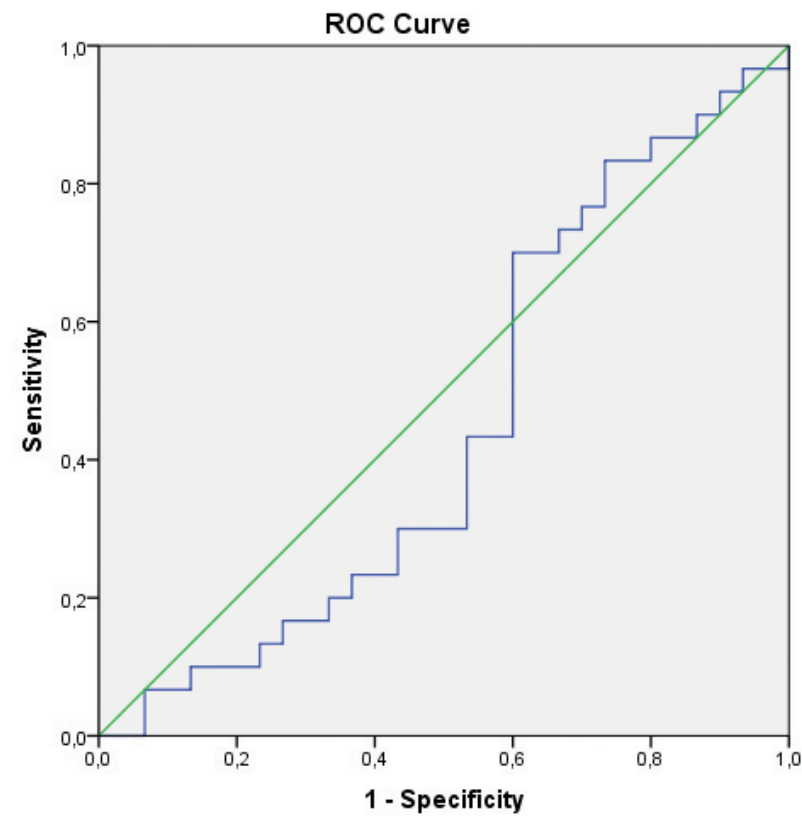

Figure 2. ROC from CA 125.

and ultrasound screening are needed for early detection of ovarian cancer ${ }^{11}$. There is no clear noninvasive or biomarker test to diagnose ovarian cancer, and the majority of patients diagnosed at an advanced stage have a poor prognosis. The discovery of new ovarian cancer biomarkers for early diagnosis, prognosis, monitoring and predictive therapeutic responses can contribute to better clinical outcomes. Therefore, new biomarkers for ovarian cancer screening are needed at an early stage.

As a traditional biomarker of ovarian cancer, CA125 has been used for 20 years. An insignificant increase in CA-125 to detect early-stage ovarian cancer is associated with a high sensitivity of CA125 which also increases in endometriosis and chronic infection. CA125 also has low specificity; for example, an increasing is found in a variety of benign gynecological diseases. Among studies that met the requirements, there were 3 original studies comparing the diagnostic efficiency of KLK6 and CA125. Koh et al. found that sensitivity and specificity of KLK6 were lower than CA125, but the combination of KLK6 and CA125 showed higher diagnostic efficiency ${ }^{12}$. E1 Sherbini et al and Diamandis et al found that KLK6 serum may have an overall sensitivity that is much lower than CA125 serum. However, KLK6 serum can increase the sensitivity of CA125. In contrast, Table 5.2 showed that a significant increase in KLK6 ( $p=0.002)$ was more specific for ovarian cancer than CA125 because it did not increase in the benign tumors. Therefore, whether the diagnostic efficiency of KLK6 is superior to CA125 remains to be clarified. However, all of these studies clearly support that KLK6 can improve CA125 diagnostic efficiency ${ }^{11,13}$.

Previous studies found that KLK6 cut-offs were different. For example, Diamandis et al (2000) reported KLK6 cut off was $15 \mathrm{ng} / \mathrm{mL}$ while Yang et al (2016) reported cut off of $3.14 \mathrm{ng} 6 / \mathrm{ml}$. There were many similar studies, such as Diamandis et al (2003) with a cut off of 4.2 and $4.4 \mathrm{ng} / \mathrm{mL}$, Koh et al with $6.0 \mathrm{ng} / \mathrm{mL}, \mathrm{El}$ Sherbini et al (2011) with $3.14 \mathrm{ng} / \mathrm{mL}$, Bandiera et al (2013) with $10.79 \mathrm{ng} / \mathrm{mL}$. Finally, most of the researches agreed that the best cut off of KLK6 was $3.14 \mathrm{ng}$ $/ \mathrm{mL}^{11-15}$.

Our study showed various types of cut-offs from KLK6 that were similar to other studies, which proposed the best cut-off of $3.14 \mathrm{ng} / \mathrm{mL}(\mathrm{p}<0.05)$ to predict ovarian malignancy. Sensitivity for detecting early stage ovarian cancer; KLK6, Ca125 (50.00\% vs 70.00\%), Specificity for detecting early stage ovarian cancer; KLK6, Ca125 (100.0\% vs 33.33\%), Positive Predictive Value to detect early stage ovarian cancer; KLK6, Ca125 (100\% vs $51.22 \%)$. KLK6 serum is a new biomarker for ovarian carcinoma. This biomarker is more specific for ovarian cancer than CA-125 because elevation is not seen in benign diseases. KLK6 diagnostic 
sensitivity is lower than the diagnostic sensitivity of CA-125 in the early stage ovarian cancer group.

KLK6 serum is a new biomarker for ovarian carcinoma. This biomarker is more specific for ovarian cancer than CA-125 because elevation is not seen in benign diseases. The diagnostic sensitivity of KLK6 is lower than CA-125 in the early stage ovarian cancer group (Table 5.4.). As discussed by Jacobs et al, the sensitivity and specificity of CA125 and KLK6, and their combination still does not meet the criteria for using these markers in the population screening setting ${ }^{16}$.

Our data showed that none of these two markers would be satisfying for early ovarian cancer markers. However, the combination between CA125 and KLK6 seems to complement each other with better specificity and same sensitivity compared to CA125 alone. Possible implications of the difference in frequency distribution curves from two markers and the proportion of late stage cancer cases when choosing statistical inference tests to evaluate the differentiating ability of tumor markers.

The results of our meta-analysis used Kappa test and Mc Nemar test which aimed to see the suitability of the results between tumor markers and their clinical findings, indicating that serum KLK6 levels could help predict the presence of ovarian cancer based on AUC (AUC $=0.737)$. These data indicate that KLK6 is a useful diagnostic marker for ovarian cancer. The poor sensitivity of KLK6 clearly limits its benefits in the diagnosis of ovarian cancer. However, high specificity also makes it a potential biomarker of ovarian cancer.

KLK6 also increases more frequently at an advanced stage and with a higher degree of disease. KLK6 is a strong prognostic indicator for the patient's condition. Patients with pre-operative KLK6 above $3.14 \mathrm{ng} / \mathrm{mL}$ had worse prognosis than patients with low pre-operative KLK6. CA-125 prognostic value disappeared in multivariate analysis, while KLK6 was an independent prognostic indicator, as shown in multivariate analysis.

We responded data from Diamandis et al about the response of chemotherapy and Kaplan-Meier curves as follows: (1) Almost all patients with high pre-operative KLK6 relapsed within 6 years, and most of them died; (2) $81 \%$ patients who did not respond to chemotherapy had high preoperative KLK6. Thus, high pre-operative KLK6 identifies patients who are refractory to chemotherapy, who will experience recurrence and death. These patients must be good candidates for other clinical trials, not chemotherapy. More appropriate clinical studies are needed to deal those problems ${ }^{13}$.
The KLK6 serum is likely to originate from tumor cells because its level decreases significantly in the postoperative stage. The study of Hoffman BR which examined the prognostic value of KLK6 on ovarian tumor extracts, found KLK6 over expression in tumor cells by immune histochemistry and subsequently proofed that KLK6 intratumor concentration was also a strong prognostic indicator. Serine proteases which do not belong to the kallikrein family also have significance prognostic in ovarian cancers, including trypsin, prostacin, hepsin, and testisin. It has been known for years that many other proteolytic enzymes have prognostic value in various types of cancer. The biological mechanism for the involvement of proteolytic enzymes in the prognosis of cancer includes their ability to decrease the extracellular matrix, thereby facilitating invasion and metastasis. It seems that some family members of the human kallikrein gene are dysregulated in ovarian cancer. Thus, other members of this family will emerge as potential biomarkers of ovarian cancer. If these proteases are involved in the development of cancer, they may be suitable candidates as therapeutic targets. This possibility is worthy of further investigation ${ }^{17}$.

Yousef GM et al. showed an example of monitoring ovarian cancer patients with KLK6 serum. More detailed studies are needed to overcome the monitoring problem of patients whose tumors do not produce CA125 but still issue KLK6. We also showed that KLK6 serum did not increase significantly in breast, thyroid, testicular, gastrointestinal, prostate, and lung cancers. Thus, KLK6 serum seems to be a specific ovarian cancer biomarker ${ }^{18}$.

High specificity in KLK6 humans shows that this is a new biomarker potential for ovarian cancer. This clearly can improve the diagnostic efficiency of ovarian cancer and KLK6 can improve CA125 diagnostic accuracy. A combination of CA125 and KLK6 shows high diagnostic efficiency (increasing diagnostic sensitivity) for ovarian cancer in all stages. Because of the limitations in this meta-analysis, additional research is needed to assess the accuracy of KLK6 diagnostics in the future ${ }^{11,14,19}$.

\section{CONCLUSION}

KLK6 is a new biomarker with high specificity for the diagnosis of ovarian cancer. This can improve the diagnostic accuracy of $\mathrm{Ca} 125$ which has high sensitivity but low specificity. A combined panel of Ca125 and KLK6 showed high diagnostic efficiency for early-sta- 
ge ovarian cancer. Our study is still having some limitations, additional research is needed to assess the accuracy of KKL6 diagnostic.

Compliance with ethics requirements: The authors declare no conflict of interest regarding this article. The

\section{References}

1. Badgwell D, Bast Jr RC. Early detection of ovarian cancer. Dis Markers. 2007;23(5-6):397-410.

2. Tung CS, Wong K-K, Mok SC. Biomarker discovery in ovarian cancer. Women's Heal. 2008;4(1):27-40.

3. Duffy MJ. Proteases as prognostic markers in cancer. Clin Cancer Res. 1996;2(4):613-8.

4. Clements JA, Willemsen NM, Myers SA, Dong Y. The tissue kallikrein family of serine proteases: functional roles in human disease and potential as clinical biomarkers. Crit Rev Clin Lab Sci. 2004;41(3):265-312.

5. Diamandis EP, Yousef GM. Human tissue kallikreins: a family of new cancer biomarkers. Clin Chem. 2002;48(8):1198-205.

6. Dong $Y$, Loessner D, Irving-Rodgers $H$, Obermair A, Nicklin $\mathrm{JL}$, Clements JA. Metastasis of ovarian cancer is mediated by kallikrein related peptidases. Clin Exp Metastasis. 2014;31(1):135-47.

7. Obiezu C V, Diamandis EP. Human tissue kallikrein gene family: applications in cancer. Cancer Lett. 2005;224(1):1-22.

8. Oikonomopoulou K, Li L, Zheng Y, Simon I, Wolfert RL, Valik D, et al. Prediction of ovarian cancer prognosis and response to chemotherapy by a serum-based multiparametric biomarker panel. $\mathrm{Br} J$ Cancer. 2008;99(7):1103.

9. Rustin GJS, Bast RC, Kelloff GJ, Barrett JC, Carter SK, Nisen PD, et al. Use of CA-125 in clinical trial evaluation of new therapeutic drugs for ovarian cancer. Clin cancer Res. 2004;10(11):391926.

10. Sarojini S, Tamir A, Lim H, Li S, Zhang S, Goy A, et al. Early detection biomarkers for ovarian cancer. J Oncol. 2012;2012.

11. El Sherbini MAH, Sallam MM, Shaban EAK, El-Shalakany AH. Diagnostic value of serum kallikrein-related peptidases 6 and 10 versus CA125 in ovarian cancer. Int J Gynecol Cancer. 2011;21(4):625-32. authors declare that all the procedures and experiments of this study respect the ethical standards in the Helsinki Declaration of 1975, as revised in 2008(5), as well as the national law. Informed consent was obtained from all the patients included in the study.

12. Koh SCL, Huak CY, Lutan D, Marpuang J, Ketut S, Budiana NG, et al. Combined panel of serum human tissue kallikreins and CA-125 for the detection of epithelial ovarian cancer. J Gynecol Oncol. 2012;23(3):175-81.

13. Diamandis EP, Scorilas A, Fracchioli S, Van Gramberen M, De Bruijn $H$, Henrik A, et al. Human kallikrein 6 (hK6): a new potential serum biomarker for diagnosis and prognosis of ovarian carcinoma. J Clin Oncol. 2003;21(6):1035-43.

14. Yang $\mathrm{F}, \mathrm{Hu} Z$, Chen $\mathrm{Y}, \mathrm{Hu} \mathrm{C}$. Diagnostic value of KLK6 as an ovarian cancer biomarker: A meta-analysis. Biomed reports. 2016;4(6):681-6

15. Bandiera E, Zanotti L, Fabricio ASC, Bucca E, Squarcina E, Romani $C$, et al. Cancer antigen 125, human epididymis 4, kallikrein 6 , osteopontin and soluble mesothelin-related peptide immunocomplexed with immunoglobulin $\mathrm{M}$ in epithelial ovarian cancer diagnosis. Clin Chem Lab Med. 2013;51(9):1815-24.

16. Jacobs IJ, Menon U. Progress and challenges in screening for early detection of ovarian cancer. Mol Cell Proteomics. 2004 3(4):355-66.

17. Hoffman BR, Katsaros D, Scorilas A, Diamandis P, Fracchioli S, De La Longrais IAR, et al. Immunofluorometric quantitation and histochemical localisation of kallikrein 6 protein in ovarian cancer tissue: a new independent unfavourable prognostic biomarker. Br J Cancer. 2002;87(7):763.

18. Yousef GM, Polymeris M-E, Yacoub GM, Scorilas A, Soosaipillai A, Popalis C, et al. Parallel overexpression of seven kallikrein genes in ovarian cancer. Cancer Res. 2003;63(9):2223-7.

19. Tamir A, Jag U, Sarojini S, Schindewolf C, Tanaka T, Gharbaran $\mathrm{R}$, et al. Kallikrein family proteases KLK6 and KLK7 are potential early detection and diagnostic biomarkers for serous and papillary serous ovarian cancer subtypes. J Ovarian Res. 2014;7(1):109. 
